\title{
Office-based sperm concentration: A simplified method for intrauterine insemination therapy
}

\author{
D R Franken, $\mathrm{PhD}$ \\ Department of Obstetrics and Gynaecology, Faculty of Health Sciences, University of the Free State, Bloemfontein, South Africa
}

Corresponding author: D R Franken (frankendr@ufs.ac.za)

Background. Intrauterine insemination (IUI) could become preferred to more invasive and expensive techniques of assisted reproduction therapy (ART) and should be offered as the first choice in cases with no female factors and mild male factor subfertility. However, developing countries and especially their rural areas often lack the necessary equipment and laboratory facilities.

Objective. To describe a simplified one-step method to determine the sperm concentration range for IUI therapy.

Methods. Semen samples from 51 sperm donors were used. Following swim-up separation, the sperm concentration of the retrieved motile fraction was counted, as well as progressive motile sperm using a standardised wet preparation. The number of sperm in a $10 \mu \mathrm{L}$ droplet covered with a $22 \times 22 \mathrm{~mm}$ coverslip was counted under $400 \times$ total magnification. The observed numbers of retrieved motile sperm were divided into three groups: $<40,40-100$ and $>101$ spermatozoa as recorded per intial estimation on the wet preparation.

Results. The mean (standard deviation) estimated sperm concentration for each group compared with actual counts per Neubauer counting chamber were: estimated $<40$ sperm $(n=14)$, mean $20(8)$, Neubauer count $2.5 \times 10^{6} / \mathrm{mL}$; estimated $40-100 \mathrm{sperm}(n=14)$, mean $71(15)$, Neubauer count $16 \times 10^{6} / \mathrm{mL}$; and estimated $>100$ sperm $(n=23)$, Neubauer count $48.3(21.7) \times 10^{6} / \mathrm{mL}$.

Conclusion. The results with IUI in male subfertility cases reported by Ombelet $e t$ al. in 1995 support the concept of first-line treatment of infertility by three to four cycles of IUI therapy in selected cases.

S Afr Med J 2015;105(4):295-297. DOI:10.7196/SAMJ.8944

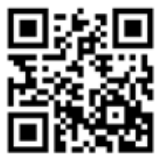

Three levels of reproductive healthcare are currently available: $(i)$ a large number of primary centres/clinics performing initial inexpensive diagnostic fertility assessments, including a basic semen analysis; (ii) a smaller number of intermediary practices that offer screening and essential reproductive healthcare treatments; and (iii) tertiary care centres and private institutions providing advanced assisted reproductive technologies in an established academic setting. ${ }^{[1]}$

Treatment for the infertile couple in a First-World setting developed rapidly through access to high-technology procedures and equipment, ready-made culture media and the finest monitoring systems in a controlled environment. However, a very different scenario exists in developing countries where clinicians manage 
Table 1. Comparison between estimated sperm count and Neubauer counting chamber recordings

\begin{tabular}{|c|c|c|c|c|c|c|}
\hline & \multicolumn{2}{|c|}{$\begin{array}{l}\text { Group 1, estimated count } \\
\quad<40 \text { sperm }(N=14)\end{array}$} & \multicolumn{2}{|c|}{$\begin{array}{l}\text { Group 2, estimated count } \\
40-100 \text { sperm }(N=14)\end{array}$} & \multicolumn{2}{|c|}{$\begin{array}{l}\text { Group 3, estimated count } \\
>101 \text { sperm }(N=23)\end{array}$} \\
\hline & Estimated count & $\begin{array}{l}\text { Neubauer count, } \\
\times 10^{6} / \mathrm{mL}\end{array}$ & Estimated count & $\begin{array}{l}\text { Neubauer count, } \\
\times 10^{6} / \mathrm{mL}\end{array}$ & Estimated count & $\begin{array}{l}\text { Neubauer count } \\
\times 10^{6} / \mathrm{mL}\end{array}$ \\
\hline Mean & 20.0 & 2.5 & 71 & 16.0 & 100.0 & 48.3 \\
\hline $\mathrm{SD}$ & 8.0 & 1.2 & 15.3 & 10.4 & 0.0 & 21.7 \\
\hline Range & $8.0-34$ & $1-4$ & $15-90$ & $6-34$ & 100 & $21-98$ \\
\hline
\end{tabular}

Table 2. Semen parameters of andrology referrals classified according to the initial estimated sperm count

\begin{tabular}{lllll}
\hline & Estimated count & Neubauer count $\times \mathbf{1 0} / \mathbf{m L}$ & Progressive motility & Morphology, \% normal \\
\hline Group 1: Estimated count $<40$ sperm & & & & 3.7 \\
Mean & 20.0 & 2.5 & 46.8 & 2.6 \\
SD & 8.0 & 1.2 & 12.0 & $1-8$ \\
Range & $8-34$ & $1-4$ & $30-65$ & 7.9 \\
Group 2: Estimated count $40-100$ sperm & & & 3.1 \\
Mean & 69.6 & 14.2 & 48.2 & $3-15$ \\
SD & 14.9 & 9.4 & 9.7 & 11.1 \\
Range & $40-89$ & $6-34$ & $40-70$ & 2.4 \\
Group 3: Estimated count $>100$ sperm & & 48.0 & 58.7 & $7-15$ \\
Mean & 99.6 & 22.1 & 11.1 & $40-80$ \\
SD & 2.1 & $21-98$ & &
\end{tabular}

an office-based infertility counselling service. In the rural regions the consulting clinician often has only the most basic equipment available to assist with diagnostic and therapeutic procedures. As part of a 'basic fertility' work-up, a simple semen analysis is therefore mandatory for couples seeking fertility treatment in a developing country. ${ }^{[2]}$ Many cases can be resolved with intrauterine insemination (IUI), as the infertility is often due to treatable reproductive tract infections. ${ }^{[3-5]}$ The interesting question arises as to whether it is possible to simplify the laboratory environment and procedures without substantially compromising the results. ${ }^{[2]}$

In cases where IUI therapy is advised as a first line of treatment, the consulting clinician can determine its feasibility by obtaining the sperm concentration and progressive motility values of the semen sample after a sperm preparation process. Ombelet et al. ${ }^{[6]}$ concluded that $>300 \times 10^{5}$ sperm with $10 \%$ progressive motility after preparation is sufficient to be used successfully in IUI cycles. The percentage of normal sperm did not play a role during their observations.

This study aimed to develop a simplified method to evaluate the sperm concentration using an office-based microscope to record sperm concentration by estimating the number of sperm on a standardised wet preparation.

\section{Methods}

Semen samples from 51 sperm donors were used in the study. Institutional review board approval was obtained. Following liquefaction, all samples were analysed according to the 2010 World Health Organization (WHO) semen analysis manual. ${ }^{[7]}$

Motile fractions were retrieved from all samples using the direct swimup technique. One $\mathrm{ml}$ of semen was placed in a sterile $15 \mathrm{ml}$ conical centrifuge tube and gently layered with $1 \mathrm{ml}$ Ham's F1-10 culture medium (supplemented with $0.3 \% \mathrm{~g}$ bovine serum albumin). The tube was placed at an angle of $45^{\circ}$ at $37^{\circ} \mathrm{C}$ for 1 hour, after which the uppermost $1 \mathrm{ml}$ of medium, which contains the motile fraction, was removed.

Wet preparations of the motile fractions were prepared by removing a $10 \mu \mathrm{l}$ aliquot, which was placed onto a clean glass slide and covered with a $22 \times 22 \mathrm{~mm}$ coverslip. The semen drop was allowed to settle out of suspension for 5 minutes. Motility was recorded as follows: progressive motility - spermatozoa moving actively, either linearly or in a large circle, regardless of speed; non-progressive motility - all other patterns of motility with an absence of progression, e.g. swimming in small circles, the flagellar force hardly displacing the head, or if only a flagellar beat could be observed; and immotile - no movement.

Following the motility evaluation, the sperm concentration was estimated on the same slide under $400 \times$ total magnification according to the WHO guidelines to determine the dilution factor before counting with a Neubauer counting chamber. The semen samples were then diluted and loaded into the Neubauer counting chamber. In cases where $>40-100$ and $>100$ sperm were observed on the wet preparation, a 1:20 dilution was used, while a 1:2 dilution was used for counts $\leq 40$. The prepared dilutions were loaded onto the two counting chambers on the Neubauer chamber. Both chambers were counted according to WHO guidelines.

The estimated sperm counts and motility values of each sample were subdivided into three groups: $<40$ sperm (group 1), 40 - 100 sperm (group 2 ), and $>101$ sperm (group 3). The estimated counts were compared with the counts recorded with the Neubauer counting chamber.

\section{Results}

The mean values (standard deviation (SD)) of the estimated sperm concentration as determined on the wet preparation, and the actual 
sperm concentration per Neubauer counting chamber, are presented in Table 1.

The mean values (SD) of the estimated Neubauer counts, progressive motility and morphology are set out in Table 2 . In all three estimated categories, the progressive motility was $>30 \%$ with mean concentrations $>1 \times 10^{6} / \mathrm{mL}$.

\section{Discussion}

The success and usefulness of ART techniques in treating infertility are well established, but the high cost of ART and lack of health insurance subsidies place a heavy burden on the couple seeking treatment for infertility. The cost of in vitro fertilisation (IVF) and intracytoplasmic sperm injection is significantly higher than the cost of a single ovarian stimulation/IUI cycle. ${ }^{[4,8]}$ Affordable low-cost office-based infertility treatment has become a field of interest among clinicians. ${ }^{[9]}$ Recent results from a pilot study reported the first pregnancies using a simplified laboratory method for human IVF. ${ }^{[10,11]}$ In the case of unexplained moderate male factor infertility, provided tubal patency has been documented, IUI with the husband's semen in natural cycles or after clomiphene citrate (CC) stimulation can be promoted as a first-line treatment without major costs or expensive infrastructure. ${ }^{[6,12]}$ The results of IUI in male subfertility cases reported by Ombelet $e t$ al..$^{[6]}$ support the concept of first-line treatment, namely three to four cycles of IUI therapy.

The method described allows the consulting clinician to establish the concentration and motility of semen to ascertain whether the sample is adequate to be used in IUI. He or she does not need expensive laboratory equipment, as a microscope, microscope glass slides, coverslips and a micropipette suffice. However, this only holds true in cases where no female factors are present. Estimating the sperm concentration should never be regarded as replacement of the standardised method prescribed in the 2010 WHO manual. ${ }^{[7]}$ The above method is recommended to clinicians in rural areas of developing countries who are confronted by childless couples who are not able to travel to distant fertility centres or cannot afford the expensive treatment that ART typically involves.

The results of this study underline that cost-effective semen analysis is possible. The mean sperm concentrations per Neubauer counting chamber were $>1 \times 10^{6} / \mathrm{mL}$ with progressive motility of $>30 \%$ in all three estimated categories, indicating that these samples could theoretically be suitable for three to four IUI cycles. In a large retrospective analysis of patients with normal ovarian response to CC stimulation, Ombelet $e t$ al.$^{[13]}$ showed overall cycle fecundity and a baby take-home rate of $14.6 \%$ and $9.9 \%$, respectively, provided the insemination motile concentration of sperm was $>1$ million. IUI remains successful in cases with $<1$ million motile spermatozoa, provided the sperm morphology score using strict criteria is $\geq 4 \%$ (cumulative pregnancy rate of $21.9 \%$ after three IUI cycles). ${ }^{[13]}$

The development of low-cost ART which is associated with a low complication rate is needed if institutions in developing countries are to be convinced to fund infertility clinics. IVF procedures can be modified to make them affordable. Studies on simplified, low-cost diagnostic procedures and ART techniques are urgently required in a low-cost setting. ${ }^{[14]}$

\section{References}

1. Huyser C. Affordable ART services in Africa: Synthesis and adaptation of laboratory services. ESHRE Monographs 2008;2008(1):77-84. [http://dx.doi.org/10.1093/humrep/den139]

2. Ombelet W, Campo R. Affordable IVF for developing countries. Reprod Biomed Online 2007;15(3):257265. [http://dx.doi.org/10.1016/S1472-6483(10)60337-9]

3. Leke RJ. The prevalence of infertility and its preventive measures in sub-Saharan Africa. In: SekaddeKigondu C, Chikamata D, Franken D, eds. Management of Infertility in AFRO \& EMRO Countries. Geneva: World Health Organization, 2002:79-91.

4. Comhaire F. Economic strategies in modern male subfertility treatment. Hum Reprod 1995;10(Suppl 1):103-106. [http://dx.doi.org/10.1093/humrep/10.suppl_1.103]

5. Nygren K, Zegers-Hochschild FZ. Documentation of infertility prevalence, treatment access and treatment outcomes in developing countries. ESHRE Monographs 2008;2008(1):5-7. [http://dx.doi. org/10.1093/humrep/den218]

6. Ombelet W, Puttermans P, Bosman E. Intrauterine insemination: A first-step procedure in the algorithm of male subfertility treatment. Hum Reprod 1995;10(Suppl 1):90-102. [http://dx.doi. org/10.1093/humrep/10.suppl_1.9]

7. World Hwealth Organization. WHO Laboratory Manual for the Examination and Processing of Human Semen. 5th ed. Geneva: WHO, 2010.

8. Peterson CM, Hatasaka HH, Jones KP, et al. Ovulation induction with gonadotropins and intrauterine insemination compared with in vitro fertilization and no therapy: A prospective nonrandomized cohort study and meta-analysis. Fertil Steril 1994;62(3):535-544.

9. Gentis RK, Siebert I, Kruger TF, de Beer ML. Implementation of an office based semen preparation method (SEP-D kit) for intra-uterine insemination (IUI): A controlled randomized study to compare the IUI pregnancy outcome between a routine (swim-up) and SEP-D Kit method. S Afr J Obstet Gynaecol 2012;18(2):54-55

10. Pilcher H. Fertility on a shoestring. Nature 2006;442:976-977. [http://dx.doi.org/10.1038/442975a]

11. Van Blerkom J, Ombelet W, Klerkx E, et al. First births with a simplified culture system for clinical IVF and embryo transfer. Reprod Biomed Online 2014;28(3):310-320. [http://dx.doi.org/10.1016/j. rbmo.2013.11.012]

12. Ombelet W, Deblaere K, Bosmans E, et al. Semen quality and intrauterine insemination. Reprod Biomed Online 2003;7(4):485-492. [http://dx.doi.org/10.1016/S1472-6483(10)61894-9]

13. Ombelet $\mathrm{W}$, Vandeput $\mathrm{H}$, van de Putte $\mathrm{G}$, et al. Intrauterine insemination after ovarian stimulation with clomiphene citrate: Predictive potential of inseminating motile count and sperm morphology? Hum Reprod 1997;12(7):1458-1463. [http://dx.doi.org/10.1093/humrep/12.7.1458]

14. Ombelet W, Cooke I, Dyer S, Serour G, Devroey P. Infertility and the provision of infertility medical Ombeles in developing countries. Hum Reprod Update 2008;14(6):605-621. [http://dx.doi.org/10.1093/
servicis services in develop
humupd/dmn 042$]$

Accepted 13 February 2015. 